\title{
Accurate Predictions for Heavy Quark Jets
}

\author{
Giulia Zanderighi \\ CERN - Theory Division, Geneve 23, CH-1211.
}

\begin{abstract}
Heavy-flavour jets enter many of today's collider studies, yet NLO predictions for these quantities are subject to large uncertainties, larger than the corresponding experimental errors. We propose a new, infrared safe definition of heavy-quark jets which allows one to reduce theoretical uncertainties by a factor of three.
\end{abstract}

\section{Introduction}

When looking at the current comparison between the inclusive $b$-jet spectra measured by $\mathrm{CDF}$ and the corresponding next-to-leading order (NLO) predictions, Fig. 1 [2], one notices two striking features. Firstly, one sees a tension between data and theory: the ratio of data over NLO is around 1.2-1.5 over the whole range of accessible transverse momenta $p_{t}$ of the jets. Secondly, one notices that the uncertainties associated with the theoretical predictions are embarrassingly large $(\sim 40-50 \%)$ for a NLO calculation and in particular they are larger than the corresponding experimental uncertainties. To understand why this happens it is useful to examine Fig. 2.

The top plots show that the large uncertainty is associated with very large $K$-factors. The middle plots confirm that the uncertainty is the same both with MCFM [3] and MCNLO [4]. Finally, the bottom plots illustrate the origin of the poor convergence of the perturbative expansion: when breaking down the Herwig [5] $b$-jet spectrum into the hard underlying channels it turns out that two NLO channels, flavour excitation, where a $b$-quark is kicked out of the sea-quarks, and gluon splitting, where a gluon in the final state splits into a $b \bar{b}$ pair, are larger than the leading order heavy quark production mechanism, flavour creation, when two incoming light partons produce a heavy quark pair.

The reason why supposedly higher order contributions are actually larger than the leading order channel can be clarified by counting soft and collinear logarithms associated with the splitting of gluons into $b \bar{b}$-pairs. It turns out that flavour excitation contributes with $\left(\alpha_{s} \ln p_{t} / m_{b}\right)^{n}$ and gluon splitting contributes with $\left(\alpha_{s} \ln p_{t} / m_{b}\right)^{2 n-1}$ relative to the leading order, $\mathcal{O}\left(\alpha_{s}^{2}\right)$ process. Since $m_{b} \ll p_{t}$ these contributions are enhanced. Moreover, the

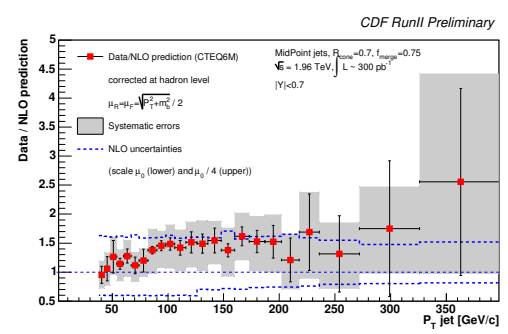

Figure 1: Ratio of the measured inclusive $b$-jet spectrum to the NLO prediction. The measurement is performed for jets with transverse momentum $38 \mathrm{GeV}<P_{T \text {,jet }}<400 \mathrm{GeV}$ and rapidity $\left|y_{\text {jet }}\right|<0.7$. 

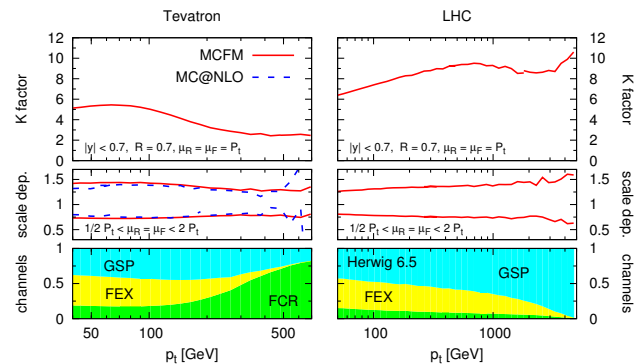

Figure 2: Top: $K$-factor for inclusive $b$-jet spectrum as computed with MCFM, clustering particles into jets using the $k_{t}$ jet-algorithm with $R=0.7$, and selecting jets in the central rapidity region $(|y|<0.7)$. Middle: scale dependence obtained by simultaneously varying the renormalisation and factorisation scales by a factor two around $p_{t}$, the transverse momentum of the hardest jet in the event. Bottom: breakdown of the Herwig inclusive $b$-jet spectrum into the three major hard underlying channels contributions (for simplicity the small $b b \rightarrow b b$ is not shown).

dominant contribution to the b-jet spectrum comes from jets originated from gluon splitting, which do not correspond to one's intuitive physical idea of a b-jet, one where a hard $b$ is produced directly in the hard scattering. ${ }^{a}$ In the following we suggest to adopt a different jet-clustering algorithm to reconstruct $b$-jets. One that by making explicit use of the flavour information eliminates all higher-order logarithmic enhancements associated to gluon splittings in the $b$-jet spectra. This means that, after resumming initial state collinear logarithms into $b$-pdfs, $b$-jets can be computed using massless QCD calculations [6] as long as one neglects power corrections $m_{b}^{2} / p_{t}^{2}$ (potentially log-enhanced).

\section{The heavy-quark jet algorithm}

We summarize here the inclusive heavy-flavour jet algorithm for hadron-hadron collisions [7]. For any pair of final-state particles $i, j$ define a class of distances $d_{i j}^{(F, \alpha)}$ parametrized by $0<\alpha \leq 2$ and a jet radius $R$

$$
d_{i j}^{(F, \alpha)}=\frac{R_{i j}^{2}}{R^{2}} \times \begin{cases}\max \left(k_{t i}, k_{t j}\right)^{\alpha} \min \left(k_{t i}, k_{t j}\right)^{2-\alpha}, & \text { softer of } i, j \text { flavoured, } \\ \min \left(k_{t i}^{2}, k_{t j}^{2}\right), & \text { softer of } i, j \text { flavourless, }\end{cases}
$$

where $R_{i j}^{2}=\Delta y_{i j}^{2}+\Delta \phi_{i j}^{2}, \Delta y_{i j}=y_{i}-y_{j}, \Delta \phi_{i j}=\phi_{i}-\phi_{j}$ and $k_{t i}, y_{i}$ and $\phi_{i}$ are respectively the transverse momentum, rapidity and azimuth of particle $i$, with respect to the beam. For each particle $i$ define a distance with respect to the beam $B$ at positive rapidity,

$$
d_{i B}^{(F, \alpha)}= \begin{cases}\max \left(k_{t i}, k_{t B}\left(y_{i}\right)\right)^{\alpha} \min \left(k_{t i}, k_{t B}\left(y_{i}\right)\right)^{2-\alpha}, & i \text { is flavoured, } \\ \min \left(k_{t i}^{2}, k_{t B}^{2}\left(y_{i}\right)\right), & i \text { is flavourless, }\end{cases}
$$

with

$$
k_{t B}(y)=\sum_{i} k_{t i}\left(\Theta\left(y_{i}-y\right)+\Theta\left(y-y_{i}\right) e^{y_{i}-y}\right)
$$

\footnotetext{
${ }^{a}$ We recall that according to the current experimental definition of a $b$-jets, a $b$-jet is any jet containing at least one $b$.
} 

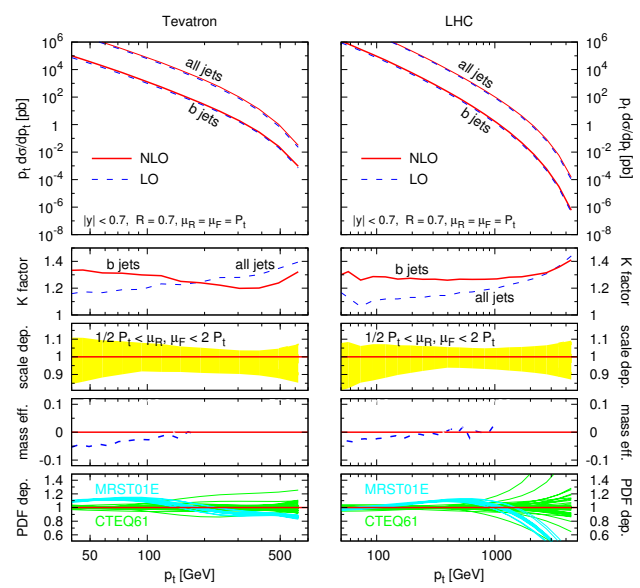

Figure 3: Inclusive jet spectrum at the Tevatron (right) and at the LHC (left). The top two panels show results for both $b$-jets and all-jets, while the lower three panels apply only to $b$-jets. See text for further details.

Similarly define a distance to the beam $\bar{B}$ at negative rapidity by replacing $k_{t B}$ in eq. (2) with $k_{t \bar{B}}$

$$
k_{t \bar{B}}(y)=\sum_{i} k_{t i}\left(\Theta\left(y-y_{i}\right)+\Theta\left(y_{i}-y\right) e^{y-y_{i}}\right) .
$$

Identify the smallest of the distance measures. If it is a $d_{i j}^{(F, \alpha)}$, recombine $i$ and $j$ into a new particle, summing their flavours and 4-momenta; if it is a $d_{i B}^{(F, \alpha)}\left(\right.$ or $\left.d_{i \bar{B}}^{(F, \alpha)}\right)$ declare $i$ to be a jet and remove it from the list of particles. Repeat the procedure until no particles are left. We define the $b$-flavour or generally the heavy-flavour of a (pseudo)-particle or a jet as its net heavy flavour content, i. e. the total number of heavy quarks minus heavy anti-quarks.

The IR-safety of this algorithm was proved in [7]. Apart from allowing one to take the limit $m_{Q}^{2} \rightarrow 0$ for the heavy quark mass (as long as collinear singularities associated with incoming heavy quarks are factorized into a heavy quark PDF), it ensures that one obtains the same results whether one considers heavy-quark flavour at parton level, or heavy-meson flavour at hadron level, modulo corrections suppressed by powers of $\Lambda_{Q C D} / p_{t}$.

\section{Results}

Our results are summarized in fig. 3 [8] where we show the inclusive $b$-jet $p_{t}$-spectrum as obtained with the flavour algorithm specified above with $\alpha=1$, and $R=0.7$, the latter having been shown to limit corrections associated with the non-perturbative underlying event [9]. The left (right) column of the figure shows results for the Tevatron Run II (LHC). We have selected only those jets with rapidity $|y|<0.7$. We also show the full inclusive jet spectrum (all jets) as obtained with a standard inclusive $k_{t}$-algorithm [10] with $R=0.7$.

We notice the considerable reduction of $K$-factors, which are around 1.3 and the moderate uncertainties associated with scale variation, signaling that the perturbative expansion is now well under control. Our predictions constitute therefore the first accurate predictions for inclusive heavy quark jets. 
We remark that very similar results are obtained when considering charmed jet spectra. An interesting issue there is that predictions are very sensitive to possible intrinsic charm components of the proton [11]. This means that this type of observable has a potential to set constraints on such intrinsic components.

A last remark concerns the feasibility of the experimental measurement of heavy flavour jets defined with our flavour algorithm. Our jet-clustering algorithm requires that one identify heavy-flavoured particles and that one uses a different distance measure when clustering heavy or light objects according to eq. (1). It is particularly important to identify cases when both heavy flavoured particles are in the same jet, so as to label this jet a gluon jet and eliminate it from the $b$-jet spectrum. Experimentally techniques for double $b$-tagging in the same jet already exist [12] and steady progress is to be expected in the near future [13]. However one has always a limited efficiency for single $b$ tagging, and even more for double $b$-tagging in the same jet. On the other hand preliminary studies indicate that one does not necessarily need high efficiencies, but what is more crucial is that one understand those efficiencies well [8]. We look forward to further investigation in this direction.

\section{Acknowledgments}

This work is done in collaboration with Andrea Banfi and Gavin Salam.

\section{References}

[1] Slides: http://indico. cern. ch/contributionDisplay $\cdot$ py? contribId=175\&sessionId=6\&conf Id=9499

[2] CDF Collaboration, Note 8418.

[3] http://mcfm.fnal.gov/

[4] S. Frixione and B. R. Webber, JHEP 0206 (2002) 029; S. Frixione, P. Nason and B. R. Webber, JHEP 0308 (2003) 007.

[5] G. Marchesini, B. R. Webber, G. Abbiendi, I. G. Knowles, M. H. Seymour and L. Stanco, Comput. Phys. Commun. 67 (1992) 465.

[6] Z. Nagy, Phys. Rev. Lett. 88, 122003 (2002); Phys. Rev. D 68, 094002 (2003).

[7] A. Banfi, G. P. Salam and G. Zanderighi, Eur. Phys. J. C 47 (2006) 113.

[8] A. Banfi, G. P. Salam and G. Zanderighi, arXiv:0704.2999 [hep-ph].

[9] A. Abulencia [CDF - Run II Collaboration], arXiv:hep-ex/0701051.

[10] S. Catani, Y. L. Dokshitzer, M. H. Seymour and B. R. Webber, Nucl. Phys. B 406, 187 (1993); S. D. Ellis and D. E. Soper, Phys. Rev. D 48 (1993) 3160.

[11] J. Pumplin, H. L. Lai and W. K. Tung, arXiv:hep-ph/0701220.

[12] D. Acosta et al. [CDF Collaboration], Phys. Rev. D 71 (2005) 092001.

[13] Andrea Bocci, "Jet flavour tagging with the CMS experiment", CMS TS-2006/004 ; Christian Weiser, "A Combined Secondary Vertex Based B-Tagging Algorithm in CMS", CMS NOTE-2006/014; M. Sapinski, "Expected performance of ATLAS for measurements of jets, b-jets, tau-jets, and ETmis", SN-ATLAS2002-012, ATL-COM-CONF-2001-006; J. Bastos, arXiv:physics/0702041. 